\section{Associations of anterior accessory or thigh posterior tributary and great saphenous reflux patterns in early stages of chronic venous valvular insufficiency}

\author{
Carlos Alberto Engelhorn, ${ }^{1,2}$ \\ Ana Luiza Dias Valiente Engelhorn,, \\ Sergio Xavier Salles-Cunha, ${ }^{2}$ \\ Carolina B. Andreatta, \\ Daniella B. Santos, ${ }^{1}$ \\ Gustavo T.M. Nakata, ${ }^{1}$ \\ Fernando A. Haida ${ }^{1}$ \\ 'Pontifícia Universidade Católica do \\ Paraná, School of Medicine; \\ ${ }^{2}$ Angiolab-Laboratório Vascular Não \\ Invasivo, Curitiba, PR, Brazil
}

\section{Abstract}

Varicose veins are a common disorder. Extensive ultrasound (US) mapping of lower extremity chronic venous valvular insufficiency includes the great saphenous vein (GSV), anterior accessory saphenous vein (AASV) and thigh posterior tributary to GSV such as the posterior accessory saphenous vein (PTSV, PASV).

The aim of this study was to determine associations between GSV-AASV-PTSV (including PASV) reflux in a specific sample population of Southern Brazilian women, mostly euro descendents, with telangiectasias, reticular veins, varices and/or intermittent edema.

US performed in 1016 extremities of 513 women, $43 \pm 18$ (18-81) years old were included. Women with previous venous thrombosis, surgery, suspicion of pelvic congestion syndrome, and men were excluded. Small saphenous vein and related thigh veins were excluded from analysis. GSV-AASV-PTSV reflux patterns were analyzed; prevalence was compared using $\chi^{2}$ statistics.

Reflux prevalence in AASV and/or thigh PTSV was 5.8\% (59/1016): $1.3 \%$ at PTSV $(n=13)$ and $4.5 \%$ at AASV $(n=46)$, significantly lower than GSV reflux: $72 \%(n=727)$ $(\mathrm{P}<0.001)$. AASV and/or PTSV reflux was associated with GSV reflux $(81 \%, \mathrm{n}=48 / 59)$; common pattern was diffuse reflux, starting at AASV/PTSV saphenous junctions (56\%, $\mathrm{n}=33 / 59$; otherwise, short, non-diffuse reflux was noted in part of the AASV/PTSV evaluated. Isolated AASV or PTSV reflux was rare (1\%, $\mathrm{n}=11 / 1016): 9$ at the AASV, and 2 at the PTSV.

US mapping of AASV/PTSV in early stages of disease, in women without pelvic congestion syndrome, increased reflux detection by $1 \%$, and improved definition of reflux patterns in about $6 \%$ of the extremities.

\section{Introduction}

Varicose veins and telangiectasias, associated with chronic venous valvular insufficiency (CVVI) of lower extremities, are common and deserving special attention. ${ }^{1-12}$ CVVI is a common subset of chronic venous insufficiency (CVI). Modern tendency is to become more specific on phlebologic investigation. The focus of this work was on CVVI exclusively, to avoid confusion with thrombosis or malformations. The following paragraphs summarize the focus on early CVVI, introduce our great saphenous vein (GSV) reflux findings in early stages of disease, and questions if we should evaluate the anterior accessory saphenous vein (AASV) and thigh posterior tributaries (PTSV) such as the posterior accessory saphenous vein (PASV) in patients with aesthetics more so than pathophysiological conditions.

An international consensus created the clinical, etiological, anatomic and pathophysiological classification (CEAP). ${ }^{10}$ CEAP has improved description of populations studied but investigation of specific subgroups is needed. We have focused on women with early stage. ${ }^{2,8-9} \mathrm{GSV}$ reflux has been related to its diameter. ${ }^{13}$ Patterns of saphenous vein reflux were created, improving communication with referring physicians. ${ }^{2,8-9,14}$ These patterns specified reflux sources and drainage points. Tributaries were more associated to GSV reflux than perforating veins in C1-C2 women. ${ }^{14,15}$ Most GSV reflux in C2 women was segmental, from one tributary to another ${ }^{2,9}$ GSV reflux was mostly in the leg, rarely in the saphenofemoral junction (SFJ). ${ }^{9}$ Telangiectasias were associated to segmental saphenous vein reflux..$^{8}$ Segmental reflux, if not treated, evolved to multi-segmental saphenous reflux. ${ }^{2}$

Non-saphenous vein reflux has received its due attention. ${ }^{16}$ Reflux in gluteal, lateral or anterior thigh, or pubic veins raise suspicion of pelvic varicosities, particularly in the presence of symptoms of pelvic congestion syndrome. ${ }^{17}$ Patients with suspected pelvic varicosities were not included in this analysis.

Thigh accessory or tributaries to the GSV may be associated with saphenous or nonsaphenous reflux. Most findings already described, however, were noted in patients with long- term CVI. The present analysis focuses on ultrasound (US) of the AASV and thigh PTSV in women with early telangiectasias, reticular veins, varicose veins and/or intermittent swelling.
Correspondence: Carlos Alberto Engelhorn and Sergio Xavier Salles-Cunha, Rua José Casagrande, 1310, Bairro Vista Alegre, Curitiba, PR, CEP 80820-590 Brazil.

Tel.: (55).41.3362.0133.

E-mail: carlos.engelhorn@pucpr.br ; sallescunha@yahoo.com

Key words: venous valvular insufficiency, duplexdoppler ultrasound, great saphenous vein, anterior accessory saphenous vein, posterior accessory saphenous vein, posterior tributary vein.

Contributions: CAE, ALDVE, primary conception, primary design, data acquisition, data interpretation, data storage, manuscript critical revision, final approval of the version to be published, fully accountable; SXSC, general conception, general design, data analysis, data interpretation, drafting the work, final approval of the version to be published, fully accountable; CBA, DBS, GTMN, FAH, secondary conception, secondary design, data retrieval, manuscript revision, initial approval of manuscript, secondary accountability.

Conflict of interests: CAE and ALE own the private Angiolab, Inc, noninvasive vascular laboratory; SXSC is a research, quality assurance consultant for Angiolab, Inc.

Funding: Angiolab, Inc, Curitiba, PR, Brazil.

Conference presentation: Oral presentation, Society for Vascular Ultrasound, 2012 Society for Vascular Ultrasound (SVU) Annual Conference, National Harbor, MD, USA.

Received for publication: 23 September 2013.

Revision received: 19 May 2014.

Accepted for publication: 21 May 2014.

This work is licensed under a Creative Commons Attribution 3.0 License (by-nc 3.0).

(C) Copyright C.A. Engelhorn et al., 2014

Licensee PAGEPress, Italy

Veins and Lymphatics 2014; 3:1938

doi:10.4081/vl.2014.1938

\section{Materials and Methods}

Patients were referred by a variety of specialists to an ISO accredited noninvasive vascular laboratory, Angiolab-Curitiba, of a major city in Southern Brazil. Venous duplex examinations were pre-approved by insurance companies. A data base has been maintained prospectively. Retrospective analysis of GSV, AASV and PTSV patterns of reflux was performed. This project was part of protocol number 207-0084-000111 of the National Commission on Ethics of Research and approved by the Ethics Research Committee of Pontificia Universidade Católica do Paraná. 


\section{Inclusion criteria}

Women with telangiectasias or reticular veins (CEAP clinical class $\mathrm{Cl}$ ), varicose veins (C2), or intermittent swelling (suggested C3A versus constant $\mathrm{C} 3$ edema) were candidates for the study. Etiology (E) was primary; anatomy (A) included superficial veins of the thigh; and pathophysiology (P) was reflux only. Data entry stopped when over 1000 extremities were posted.

\section{Exclusion criteria}

Exclusion of all men avoided gender variability. Women with constant, chronic edema, skin changes, and healed or open ulcers ( $\mathrm{C} 3$, C4A, C4B, C5, C6), history of previous deep venous thrombosis (DVT), suspicion of pelvic venous insufficiency, venous malformations, and/or previous direct treatment of lower extremity veins were excluded. Exclusion based on treatment included surgery, minimally invasive thermal ablation, and chemical ablation with foam or sclerosant.

Venotonic oral medication, or compression stockings, or deep venous reflux due to valvular insufficiency only were not reason for exclusion.

Small saphenous vein (SSV), respective thigh extensions, and other leg veins besides GSV were not analyzed.

\section{Patient population}

US data from 1016 limbs of 513 women, $43 \pm 13$ (range 18-81) years-old, were analyzed. Patients represented an educated, middle class of Southern Brazil, mostly European descendants, from Portuguese, Spanish, Italian, German, and Ucranian ancestry. This sample population did not represent the Brazilian multi-ethnicity, neither the various social classes. Specifically, rural or community groups were not represented. The women evaluated had aesthetic and/or functional interests; visual appearance, itching or slight burning sensation were common causes to seek medical attention. Clinical presentations C1C2 and suggested-C3A, intermittent swelling, could be combined, varying from almost none to easily detectable.

\section{Ultrasonographic duplex-Doppler examination}

International recommendations were followed and adapted to cases of early stage venous valvular insufficiency. . $^{18,19}$ US examinations were performed and interpreted by physicians certified in vascular noninvasive testing. Patients received instructions prior to and during the examination. Siemens (Issaquah, WA, USA) Elegra or Antares scanners were employed. The most commonly used transducer was centered at $7 \mathrm{MHz}(4-9 \mathrm{MHz})$. DVT, venous obstruction, and/or malformations were ruled out with the patient supine or standing. Superficial venous mapping was performed in the standing position. Patient movement and periods of rest avoided fainting. Examinations lasted around $40 \mathrm{~min}$. Valsalva or Valsalva-like maneuvers such as talking and laughing were employed according to individual behavior. Muscle and venous compression maneuvers were performed manually, proximal and distally to probe placement. Backflow causing venous filling was commonly tested with multiple compression maneuvers to avoid false reflux data. Reverse flow through potentially normal, valveless, limited saphenous segments, however, was reported if a draining tributary had reflux; apparently normal draining via a perforating vein was described as reflux, particularly if a diameter dilatation was noted in such segment. Short reflux segments, as short as $4 \mathrm{~cm}$, were detectable with this compression technique. ${ }^{8}$ This approach emphasized testing variety and completeness, taking into consideration that daily life conditions were difficult to be reproduced in the vascular laboratory. Local changes in venous diameters were noted. Segmental reflux was recorded with information about source and drainage points. According to the original concept, reflux lasting less than $0.5 \mathrm{~s}$ was normal. ${ }^{20}$ Consequently reflux lasting longer than $0.5 \mathrm{~s}$ was considered not normal. Superficial vein reflux lasted longer than $1 \mathrm{~s}$ in most cases.

GSV and its tributaries were evaluated from the groin to the ankle. Although modern consensus mandates that the GSV term be used for the vein within the saphenous compartment, long, GSV reflux patterns may have included small tributary segments outside the saphenous compartment. Diffuse GSV reflux included either the GSV proper in the calf or the posteri- or arch-posterior accessory vein extending to the ankle level. In general, the SFJ defined common femoral yes-or-no source of reflux and GSV yes-or-no reflux from a source at or near the junction. The superficial epigastric vein is an example of a non-common femoral source of GSV reflux. Non common femoral reflux source, however, was expected to have low prevalence in the population studied.

GSV reflux patterns as shown in Figure 1: i) segmental if from a tributary or perforating vein distal to the SFJ to a tributary or perforating vein proximal to the ankle; segmental reflux is most common in the calf than in the thigh or knee; ${ }^{9}$ ii) distal from a tributary or perforating vein to the ankle; iii) multi-segmental with two or more distinct refluxing segments but a normal SFJ; iv) multi-segmental with reflux at the SFJ; v) proximal from the SFJ to a tributary or perforating vein at the thigh or calf above the ankle, having a normal distal segment; vi) diffuse throughout the entire vein, from SFJ to the ankle; vii) perijunction if nonfemoral-saphenous, femoro-nonsaphenous, nonfemoral-nonsaphenous reflux at the junction; or viii) no reflux.

AASV and PTSV were investigated in the thigh. The AASV drained the anterior surface of the thigh, running parallel to the GSV, into the saphenous-femoral junction. ${ }^{19,21}$ AASV alignment with the femoral artery and vein were searched for proper identification. The PTSV, including PASV, drained the posterior surface of the thigh into the proximal GSV segment. ${ }^{19,21}$ A classical, noticeable, predominant Giacomini vein from the SSV to the GSV was not part of PTSV statistics in this study. The PTSV included as such in this study drained primarily the posterior thigh. Origin and drainage of varicose veins were noted. Usual mapping included dis-

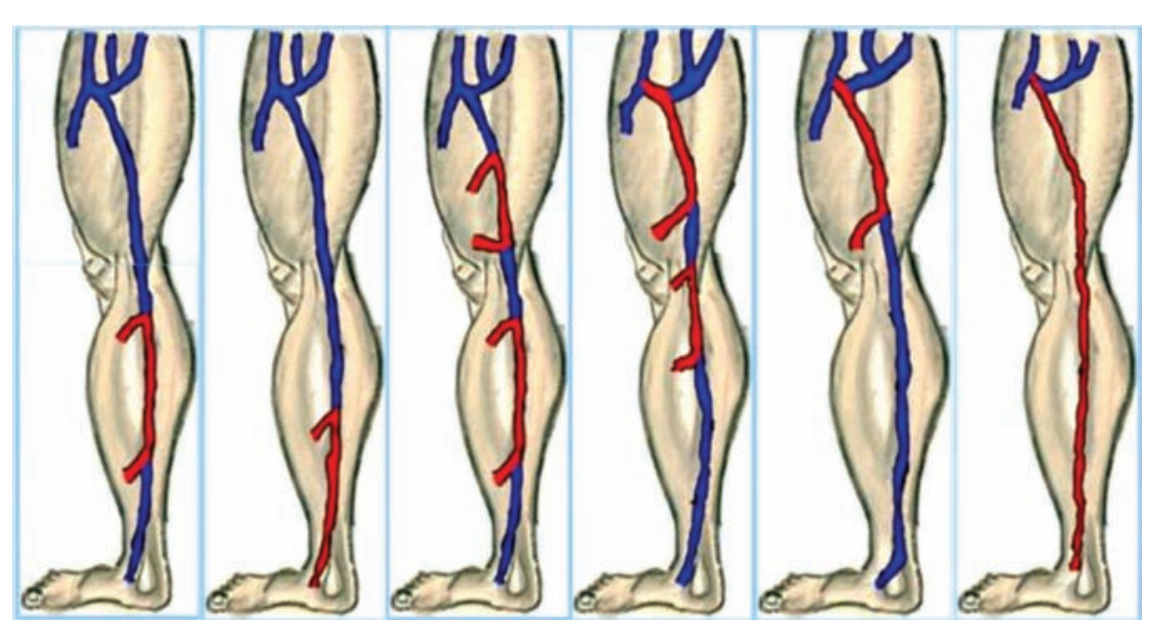

Figure 1. Diagrams exemplifying common great saphenous vein reflux patterns, from left to right of the observer: segmental, distal, multi-segmental with normal saphenofemoral junction (SFJ), multisegmental with refluxing SFJ, proximal, and diffuse throughout the vein (modified from Engelhorn et al., drawings by Monique Salles-Cunha). 
tances from source and drainage points of reflux to anatomic landmarks.

AASV and PTSV reflux were classified as diffuse or non-diffuse. Diffuse AASV reflux, throughout the vein segment studied, started at the femoral junction and continued at least to the mid-distal thigh or knee. Non-diffuse reflux occurred in segments shorter than the venous segment studied. PTSV reflux was diffuse if included the junction to the GSV and extended distally for the entire segment evaluated, usually to mid-distal thigh. PTSV reflux was non-diffuse if shorter than the venous segment evaluated. Figure $2^{19,21}$ diagrams common configurations. Figure 3 exemplifies AASV reflux at the femoral junction.

\section{Statistics}

Descriptive statistics indicated frequency or prevalence of GSV, AASV and/or PTSV reflux. Prevalence was compared using $\chi^{2}$ statistics from Excel files.

\section{Results}

This session describes patterns and prevalence of GSV, AASV and PTSV reflux, and the relations between AASV or PTSV and GSV patterns of reflux.

\section{Prevalence of great saphenous}

vein, anterior accessory saphenous vein and posterior tributary

\section{saphenous vein reflux}

All GSV were examined. There were high percentages of insignificant or undetected AASV or PTSV; AASV and PTSV were evaluated for reflux in $40 \%(406 / 1016)$ of the limbs: AASV in $34 \%$ (348/1016), a PTSV in $9 \%(88 / 1016)$, and combined in $3 \%(30 / 1016)$ of the extremities.

Reflux was most prevalent in the GSV, 72\% (727/1016), then in the AAVS, 4.5\% (46/1016), and least prevalent in PTVS, $1.3 \%$ (13/1016) ( $\mathrm{P}<0.001$ for all comparisons). Percentages of reflux per AASV or PTSV examined were 13\% (46/348) and 15\% (13/88) respectively.

Total prevalence of reflux in either GSV, AASV and/or PASV was 73\% (738/1016). Single or multiple locations of reflux were: i) GSV only: 679 limbs ( $66.8 \%$ of 1016 limbs); ii) AASV only: 9 limbs (0.9\%), 3 with diffuse and 6 with non-diffuse reflux; iii) PTSV only: 2 limbs (0.2\%) with non-diffuse reflux; iv) GSV and AASV: 37 limbs (3.6\%), 23 with diffuse and 14 with non-diffuse AASV reflux; v) GSV and PTSV: 11 limbs (1.1\%), 7 with diffuse and 4 with non-diffuse PASV reflux; vi) AASV and PASV: 0; vii) GSV, AASV and PASV: 0 limbs; and viii) no reflux in the GSV, AASV and PTSV: 278 limbs (27\%).

Non-diffuse reflux predominated in cases of isolated AASV or PTSAV reflux, 73\% (8/11) vs $27 \%(3 / 11)$ with diffuse reflux $(\mathrm{P}=0.03)$.

\section{Patterns of great saphenous vein reflux}

Prevalence of GSV flow or reflux patterns were, from most to least common: i) segmental reflux, $32.0 \%$ ( $\mathrm{n}=325 / 1016$ limbs); ii) no reflux, $28.4 \% \quad(n=289 / 1016)$; iii) multi-segmental reflux with normal SFJ, 11.8\% ( $\mathrm{n}=120 / 1016)$; iv) proximal, $8.8 \%(\mathrm{n}=89 / 1016)$; v) distal, $8.0 \%$ ( $n=81 / 1016)$; vi) multi-segmental reflux with SFJ reflux, 7.3\% ( $\mathrm{n}=74 / 1016)$; vii) diffuse reflux, 3.1\% ( $\mathrm{n}=31 / 1016)$; and viii) peri-SFJ reflux, $0.7 \%(n=7 / 1016)$.

Segmental or multi-segmental reflux patterns with normal SFJ were noted in $44 \%$

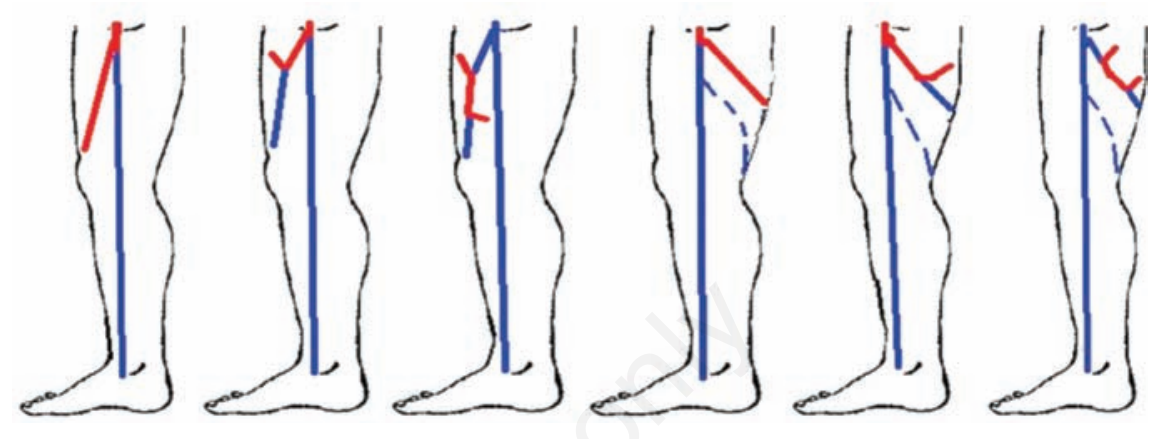

Figure 2. Diagrams exemplifying diffuse or non-diffuse reflux of the anterior accessory saphenous vein (AASV) or thigh posterior tributary to saphenous vein (PTSV) in red, located anterior or posteriorly in the thigh in relation to the great saphenous vein in blue. Identification of the AASV and PTSV were consistent with descriptions by Cavezzi et al. and Caggiati et al. ${ }^{19,21}$ Diffuse reflux was present in the entire segment evaluated. Nondiffuse reflux was limited to small vein segments, components of the entire venous segment evaluated. Short length, proximal reflux was included in the non-diffuse group. Dotted line indicating that the small to great saphenous connecting vein was not part of the PTSV analysis.

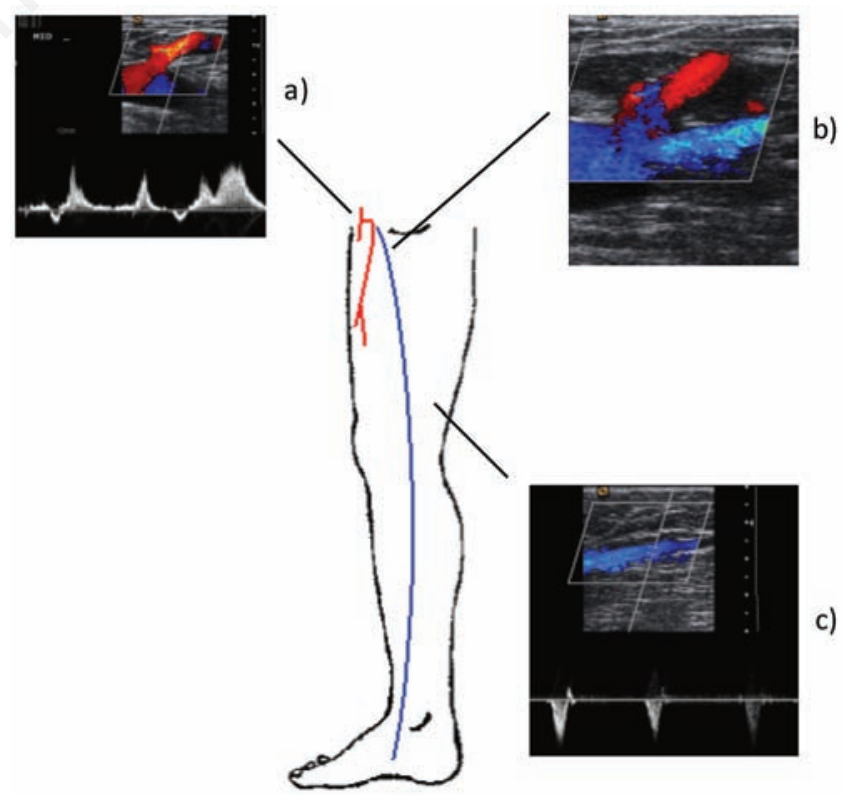

Figure 3. Duplex Doppler ultrasound showing anterior accessory saphenous vein (AASV) reflux at the femoral junction. A) Doppler reverse flow signal from the femoral to the AASV; B) color flow image of the common femoral and great saphenous vein (GSV) in blue and the AASV in red (anatomic variations are described, for example, in http://www.phlebologia.com/en/jonction_sapheno_femorale.asp); and C) color flow image of a normal GSV in blue and Doppler signal showing GSV flow toward the common femoral vein. 
( $n=445 / 1016)$ of extremities. With the addition of distal reflux, GSV reflux with normal SFJ was noted in $52 \%(n=526 / 1016)$ of limbs, equivalent to $72 \%(n=526 / 727)$ of refluxing GSV. Junction reflux (iv+vi+vii+viii) was noted in 20\% ( $n=201 / 1016)$ of limbs. SFJ reflux was significantly less common than normal SFJ flow $(\mathrm{P}<0.001)$.

\section{Patterns of anterior accessory saphenous vein reflux}

Prevalence of AASV flow or reflux patterns were, from most to least common: i) insignificant flow or size, $65.7 \%$ ( $n=668 / 1016)$; ii) no reflux, $29.7 \%(\mathrm{n}=302 / 1016)$; iii) diffuse reflux, $2.6 \%(\mathrm{n}=26 / 1016)$; and iv) non-diffuse reflux segmental away from the junction or short proximal segment, $2.0 \%(n=20 / 1016)$.

Prevalence of AASV diffuse, proximal or segmental reflux was $4.5 \%(n=46 / 1016)$.

\section{Patterns of posterior tributary to saphenous vein reflux}

Prevalence of PTSV flow or reflux patterns were, from most to least common: i) insignificant flow or size, $91.3 \%(\mathrm{n}=928 / 1016)$; ii) no reflux, 7.4\% ( $\mathrm{n}=75 / 1016)$; iii) diffuse reflux, $0.7 \%(\mathrm{n}=7 / 1016)$; and iv) non-diffuse reflux segmental away from the junction or short proximal segment, $0.6 \%(n=6 / 1016)$.

Prevalence of PTSV diffuse, proximal or segmental reflux was $1.3 \%(\mathrm{n}=13 / 1016)$.

\section{Relations between anterior accessory saphenous vein and great saphenous vein reflux patterns}

Table 1 shows cross-tabulation between patterns of reflux in the AASV and GSV. Distribution of GSV reflux patterns was significantly different as a function of AASV patterns ( $\mathrm{P}<0.001, \chi^{2}$ applied to $4 \times 8$ data of Table 1 ). Diffuse AASV reflux was associated with higher prevalence of SFJ reflux.

Some two-by-two comparisons provided the following statistics: i) diffuse AASV plus SFJ reflux showed significantly higher prevalence, $\mathrm{n}=14$ of 26 (54\%), than the expected prevalence of approximately $20 \%, \mathrm{n}=5$, as shown in Table $2(\mathrm{P}<0.001)$; and ii) non-diffuse AASV plus SFJ reflux showed a real prevalence, $n=4$ of 20 (Table 1), equal to the expected prevalence of $20 \%(\mathrm{P}=0.98)$.

\section{Relations between posterior tributary to saphenous vein and great saphenous vein reflux patterns}

Table 3 shows cross-tabulation between patterns of reflux in the PTSV and GSV. Distribution of GSV reflux patterns was not significantly different as a function of PTSV
Table 1. Cross-correlation between anterior accessory saphenous vein and great saphenous vein patterns of reflux.

\begin{tabular}{lccccccccc}
$\begin{array}{l}\text { Type } \\
\text { AASV* }\end{array}$ & Dif & MsP & Pro & PeJ & $\begin{array}{c}\text { GSV } \\
\text { Mseg }\end{array}$ & Dst & Seg & NoR & Total \\
Diffuse & 2 & 8 & 2 & 2 & 3 & 2 & 4 & 3 & 26 \\
Non-diffuse & 0 & 2 & 0 & 2 & 1 & 3 & 6 & 6 & 20 \\
\hline No reflux & 5 & 11 & 16 & 1 & 44 & 23 & 100 & 102 & 302 \\
Insignificant & 24 & 53 & 71 & 2 & 72 & 53 & 215 & 178 & 668 \\
\hline Total & 31 & 74 & 89 & 7 & 120 & 81 & 325 & 289 & 1016 \\
Percentages & & & & & & & & & \\
Diffuse & 7.7 & 30.8 & 7.7 & 7.7 & 11.5 & 7.7 & 15.4 & 11.5 & $100 \%$ \\
Non-diffuse & 0.0 & 10.0 & 0.0 & 10.0 & 5.0 & 15.0 & 30.0 & 30.0 & $100 \%$ \\
\hline No reflux & 1.7 & 3.6 & 5.3 & 0.3 & 14.6 & 7.6 & 33.1 & 33.8 & $100 \%$ \\
Insignificant & 3.6 & 7.9 & 10.6 & 0.3 & 10.8 & 7.9 & 32.2 & 26.6 & $100 \%$ \\
\hline Total & 3.1 & 7.3 & 8.8 & 0.7 & 11.8 & 8.0 & 32.0 & 28.4 & $100 \%$ \\
\hline
\end{tabular}

GSV, great saphenous vein reflux patterns; AASV, anterior accessory saphenous vein; Dif, diffuse; MsP, multisegmental - proximal, including saphenofemoral junction; Pro, proximal; PeJ, perijunction; Mseg, multisegmental; Dst, distal; Seg, segmental; NoR, no reflux.

*Diffuse AASV started at the femoral junction reflux. Non-diffuse reflux was detected only in part of the AASV examined.

Table 2. Cross-correlation between anterior accessory saphenous vein and great saphenous vein patterns of reflux.

\begin{tabular}{lccc}
$\begin{array}{l}\text { Type } \\
\text { Diffuse AASV }\end{array}$ & \multicolumn{3}{c}{ GSV-SFJ reflux* } \\
& Yes & No & Total \\
Real prevalence & & $12(46 \%)$ & $26(100 \%)$ \\
Yes & $14(54 \%)$ & $803(81 \%)$ & $990(100 \%)$ \\
No & $187(19 \%)$ & 815 & 1016 \\
\hline Total & 201 & & \\
Expected prevalence & & $21(81 \%)$ & $26(100 \%)$ \\
Yes & $5(19 \%)$ & $794(80 \%)$ & $990(100 \%)$ \\
No & $196(20 \%)$ & 815 & 1016 \\
\hline Total & 201 & & \\
\hline
\end{tabular}

GSV, great saphenous vein; SFJ, saphenofemoral junction; AASV, anterior accessory saphenous vein.

*SFJ reflux: diffuse, multisegmental, proximal or perijunctional; ㅇfffuse AASV started at the femoral junction reflux. Non-diffuse reflux was detected only in part of the AASV examined.

Table 3. Cross-correlation between thigh posterior tributary to saphenous vein and great saphenous vein patterns of reflux.

\begin{tabular}{lccccccccc}
$\begin{array}{l}\text { Type } \\
\text { PISV* }\end{array}$ & Dif & MsP & Pro & PeJ & $\begin{array}{c}\text { GSV } \\
\text { Mseg }\end{array}$ & Dst & Seg & NoR & Total \\
Diffuse & 0 & 2 & 0 & 0 & 0 & 0 & 5 & 0 & 7 \\
Non-diffuse & 0 & 0 & 0 & 0 & 0 & 0 & 4 & 2 & 6 \\
\hline No reflux & 2 & 2 & 4 & 0 & 12 & 11 & 18 & 26 & 75 \\
Insignificant & 29 & 70 & 85 & 7 & 108 & 70 & 298 & 261 & 928 \\
\hline Total & 31 & 74 & 89 & 7 & 120 & 81 & 325 & 289 & 1016 \\
\hline Percentages & & & & & & & & & \\
Diffuse & 0.0 & 28.6 & 0.0 & 0.0 & 0.0 & 0.0 & 71.4 & 0.0 & $100 \%$ \\
Non-diffuse & 0.0 & 0.0 & 0.0 & 0.0 & 0.0 & 0.0 & 66.7 & 33.3 & $100 \%$ \\
\hline No reflux & 2.7 & 2.7 & 5.3 & 0.0 & 16.0 & 14.7 & 24.0 & 34.7 & $100 \%$ \\
Insignificant & 3.1 & 7.5 & 9.2 & 0.8 & 11.6 & 7,5 & 32.1 & 28.1 & $100 \%$ \\
\hline Total & 3.1 & 7.3 & 8.8 & 0.7 & 11.8 & 8.0 & 32.0 & 28.4 & $100 \%$
\end{tabular}

GSV, great saphenous vein reflux patterns; PTSV, posterior tributary to saphenous vein; Dif, diffuse; MsP, multisegmental - proximal, including saphenofemoral junction; Pro, proximal; PeJ, perijunction; Mseg, multisegmental; Dst, distal; Seg, segmental; NoR, no reflux.

*Diffuse PTSV started at the GSV. Non-diffuse reflux was detected only in a short segment of the PTSV examined. 
patterns $\left(\mathrm{P}=0.17, \chi^{2}\right.$ applied to $4 \times 8$ data of Table 3$)$.

\section{Summary}

The population studied had a prevalence of $73 \%(738 / 1016)$ of reflux in the GSV and thigh AASV, PTSV. Reflux prevalence was $72 \%$ (727/1016) in the GSV, 4.5\% (46/1016) in the AASV, and 1.3\% (13/1016) in PTSV. Testing for AASV and PTSV reflux increased detection of reflux by $1 \%$ (11/1016) and improved definition of patterns of reflux in 5.8\% (59/1016) of the lower extremities. AASV diffuse reflux correlated with a 2.5 times higher prevalence of GSV-SFJ reflux than expected in general (54\% vs 20\%).

\section{Discussion}

We emphasized ultrasound findings in early stages of CVVI in women. CVVI emphasizes valvular insufficiency, not highlighted when the CVI expression, that includes thrombosis, is used. Attention to CVVI increased with development of minimally invasive thermal and chemical ablation treatments such as laser or radio frequency and foam sclerotherapy. ${ }^{1,3,6,7,12}$ Advances in duplex-Doppler ultrasonography have contributed to CVVI understanding, diagnosis, treatment and followup. ,8-9,13-20,22-24 $^{2}$

International consensuses occurred to minimize confusion in past phlebologic literature. ${ }^{1,5,10}$ Description of patient populations studied improved but studies of even more specific sample populations, based on gender for example, ${ }^{25}$ should be promoted.

Any venous segment could become an initial source of reflux and varicose veins. Segmental GSV reflux from a tributary source to a tributary drainage is the most common. A perforating vein source of reflux is not common finding in early stages of disease. Perforating vein drainage was considered reflux, particularly if segmental diameter changes were noted at source and drainage points. Segmental reflux propagates mostly to multi-segmental reflux if not treated; ${ }^{2}$ reflux evolves from the weakest to the next weakest point, regardless of anatomic relationships. We investigated if AASV or PTSV could be weak sites for reflux development early on. PTSV were originally interpreted as the posterior accessory saphenous vein; but this specific interpretation has been questioned leading to a more general PTSV description.

Most prevalence data represent a select clinic group and not the population in general. The sample in this study had GSV reflux with similar prevalence (72\%) as previously reported for a varicose vein $\mathrm{C} 2$ group (77\%), almost double than reported for a telangiectasia $\mathrm{Cl}$ group (44\%)., ${ }^{8,9}$ Saphenofemoral junction reflux is uncommon in early stages of disease; previously published prevalence in women with varicose veins or telangiectasias was less than $20 \%,{ }^{8,9}$ the prevalence found in the sample population of this study (Table 2). Reflux at the AASV contributed to SFJ reflux; SFJ reflux prevalence almost tripled in cases of diffuse AASV reflux (Table 2). Garcia-Gimeno described AASV reflux prevalence over $10 \%$ for a sample population with over $60 \%$ SFJ reflux, ${ }^{22}$ a group apparently with more advanced diseased than the one in this work. Determination of terminal valve patency is significant to design type of treatment. ${ }^{23}$

Brazilian custom is to treat telangiectasias and small reticular veins with $75 \%$ dextrose liquid sclerosant. Modern tendency includes foam sclerotherapy with polydocanol for relatively larger veins. Advanced technology has been implemented in clinics specialized in both symptomatic and aesthetic phlebology. ${ }^{26-29}$ Cold air sedation, laser ablation and liquid sclerosis with $75 \%$ dextrose have been combined to treat small veins, resulting in the Cryo Laser and Cryo sclerotherapy or CLaCs technique. ${ }^{26}$ This procedure is guided by a near infra red imaging Vein Viewer (Luminetx Co, Memphis, TN, USA). ${ }^{27}$ This device colors over the skin more superficial veins than noted by the human eye or detected by US. The technique denominated augmented reality shows small nutrient veins underneath telangiectasias consistently. ${ }^{28}$ Results of the CLaCs technique was reported at the 2013 International Union of Phlebology (UIP) meeting showing improvement of skin conditions with $1 \%$ of the patients complaining of hyperpigmentation. ${ }^{29}$

In this investigation, we focused on AASV and PTSV, not frequently included in CVVI studies. AASV and PTSV rarely were the initial location of reflux in C1-C2-C3A women. Our and Seidel's data suggested propensity for early reflux and varicosity abnormalities more so in the calf than in the thigh..$^{8-9,24}$ Calf veins other than the GSV were not included in this analysis. Above-knee source of SSV reflux was expected to be less than $3 \%$ in $\mathrm{C} 2$ women. ${ }^{9}$

Saphenous accessory and tributary veins in the thigh are less vulnerable to hydrostatic pressures and kinetic energies than the great saphenous and the veins in the calf. Approximately 1 in 17 extremities tested may have AASV or PTSV reflux. On another angle, 1 in 100 extremities may have only AASV or PTSV reflux. If the goal is to detect CVVI early to follow or treat patients, AASV and PTSV should be evaluated despite low reflux prevalence.

Significant changes in patterns of reflux are expected if a woman presents with pelvic congestion syndrome. ${ }^{17}$ Veins in all anatomic regions of the thigh have been evaluated if pelvic venous reflux is suspected, particularly if thigh varicosities are present. These patients, however, were excluded from this early stage of disease study. Also, the prevalence described herein cannot be applied to men in general or women $\mathrm{C} 4, \mathrm{C} 5-\mathrm{C} 6$ or even true $\mathrm{C} 3$ with constant edema.

In summary, it was reconfirmed that most women with early stages of CVVI had segmental or distal GSV reflux not involving the SFJ. Furthermore, prevalence of AASV or PTSV reflux was low, and mostly associated with GSV reflux. AASV or PTSV localized reflux, however, was the only abnormality detected in $1 \%$ of the extremities. US of these veins altered reflux pattern definition in about $6 \%$ of the extremities, and AASV diffuse reflux indicated more prevalence of SFJ reflux in the affected GSV.

\section{References}

1. Rabe E, Breu F, Cavezzi A, et al. European guidelines for sclerotherapy in chronic venous disorders. Phlebology 2013;29:33854.

2. Engelhorn CA, Manetti R, Baviera MM, et al. Progression of reflux patterns in saphenous veins of women with chronic venous valvular insufficiency. Phlebology 2012;27: 25-32.

3. Chaar CI, Hirsch SA, Cwenar MT, et al. Expanding the role of endovenous laser therapy: results in large diameter saphenous, small saphenous, and anterior accessory veins. Ann Vasc Surg 2011;25: 656-61.

4. Seidel AC, Mangolim AS, Rossetti LP, et al. Prevalence of lower limb superficial venous insufficiency in obese and non-obese patients. J Vasc Bras 2011;10:124-30.

5. Gloviczki P, Comerota AJ, Dalsing MC, et al. The care of patients with varicose veins and associated chronic venous diseases: clinical practice guidelines of the Society for Vascular Surgery and the American Venous Forum. J Vasc Surg 2011;53:2S-48S.

6. Theivacumar NS, Darwood RJ, Gough MJ. Endovenous laser ablation (EVLA) of the anterior accessory great saphenous vein (AAGSV): abolition of sapheno-femoral reflux with preservation of the great saphenous vein. Eur J Vasc Endovasc Surg 2009;37:477-81.

7. Yamaki T, Nozaki M, Sakurai H, et al. Multiple small-dose injections can reduce the passage of sclerosant foam into deep veins during foam sclerotherapy for varicose veins. Eur J Vasc Endovasc Surg 2009;37:343-8.

8. Engelhorn CA, Engelhorn AL, Cassou MF, Salles-Cunha S. Patterns of saphenous venous reflux in women presenting with lower extremity telangiectasias. 
Dermatol Surg 2007;33:282-8.

9. Engelhorn CA, Engelhorn AL, Cassou MF, Salles-Cunha SX. Patterns of saphenous reflux in women with primary varicose veins. J Vasc Surg 2005;41:645-51.

10. Eklöf B, Rutherford RB, Bergan JJ, et al. Revision of the CEAP classification for chronic venous disorders: consensus statement. J Vasc Surg 2004;40:1248-52.

11. Beebe-Dimmer JL, Pfeifer JR, Engle JS, Schottenfeld D. The epidemiology of chronic venous insufficiency and varicose veins. Ann Epidemiol 2005;15:175-84.

12. Frullini A, Cavezzi A. Sclerosing foam in the treatment of varicose veins and telangiectases: history and analysis of safety and complications. Dermatol Surg 2002;28:11-5.

13. Engelhorn C, Engelhorn A, Salles-Cunha S, et al. Relationship between reflux and great saphenous vein diameter. J Vasc Technol 1997;21:167-71.

14. Engelhorn CA, Engelhorn AL, Cassou MF, et al. Functional anatomic classification of saphenous vein insufficiency in the planning for varicose vein surgery based on color doppler ultrasound. J Vasc Bras 2004;3:13-9.

15. Engelhorn C, Engelhorn A, Casagrande C, Salles-Cunha SX. Sources and drainages of saphenous vein reflux in patients with primary varicose veins. Poster, final program of the American Venous Forum 11th Annual Meeting of the, Dana Point, CA, February 18-21, 1999, p 96.

16. Labropoulos N, Tiongson J, Pryor L, et al.
Nonsaphenous superficial vein reflux. J Vasc Surg 2001;34:872-7.

17. Barros FS, Gomez Perez JM, Zandonade E, et al. Evaluation of pelvic varicose veins using color doppler ultrasound: comparison of results obtained with ultrasound of the lower limbs, transvaginal ultrasound and phlebography. J Vasc Bras 2010;9:15-23.

18. Coleridge-Smith P, Labropoulos N, Partsch $\mathrm{H}$, et al. Duplex ultrasound investigation of the veins in chronic venous disease of the lower limbs-UIP consensus document. Part I. Basic principles. Eur J Vasc Endovasc Surg 2006;31:83-92.

19. Cavezzi A, Labropoulos N, Partsch H, et al. Duplex ultrasound investigation of the veins in chronic venous disease of the lower limbs-UIP consensus document. Part II. Anatomy. Eur J Vasc Endovasc Surg 2006;31:288-99.

20. Labropoulos N, Tiongson J, Pryor L, et al. Definition of venous reflux in lowerextremity veins. J Vasc Surg 2003;38:793-8.

21. Caggiati A, Bergan JJ, Gloviczki P, et al. Nomenclature of the veins of the lower limb: Extensions, refinements, and clinical application; International Interdisciplinary Consensus Committee on Venous Anatomical Terminology. J Vasc Surg 2005; 41:719-24.

22. García-Gimeno M, Rodríguez-Camarero S, Tagarro-Villalba $S$, et al. Duplex mapping of 2036 primary varicose veins. J Vasc Surg 2009;49:681-9.

23. Zamboni P, Gianesini S, Menegatti E, et al.
Great saphenous varicose vein surgery without saphenofemoral junction disconnection. Br J Surg 2010;97:820-5.

24. Seidel AC, Miranda F Jr, Juliano Y, et al. Prevalence of varicose veins and venous anatomy in patients without truncal saphenous reflux. Eur J Vasc Endovasc Surg 2004;28:387-90.

25. Beebe HG, Scissons RP, Salles-Cunha SX, et al. Gender bias in use of venous ultrasonography for diagnosis of deep venous thrombosis. J Vasc Surg 1995;22:538-42.

26. Miyake RK, Duarte FH, Fidelis RJ, Miyake H. New leg veins air cooled treatment using 1064nm laser combined with sclerotherapy: technique description and one year follow-up. Lasers Med Sci 2003; 18:S22.

27. Miyake RK, Zeman HD, Duarte FH, et al. Vein imaging: a new method of near infrared imaging, where a processed image is projected onto the skin for the enhancement of vein treatment. Dermatol Surg 2006;32:1031-8.

28. Miyake K. Prevalence of small varicosities among patients with or without telangiectasias on the lower limbs estimated by augmented reality examination. Int Angiol 2013;32:124-5.

29. Miyake K. Case report of 195 patients classified by duplex scanning and augmented reality and treated by cryo-laser and cryosclerotherapy: results and complications. Int Angiol 2013;32:153. 